\title{
THE COVID STONES COLLABORATIVE: HOW HAS THE MANAGEMENT OF URETERIC STONES CHANGED DURING AND AFTER THE COVID-19 PANDEMIC? RATIONALE AND STUDY PROTOCOL \\ The COVID Stones Collaborative Steering Group*
}

Fanourios Georgiades ${ }^{1 *}$, Matthew H V Byrne ${ }^{2}$, Catherine Elizabeth Lovegrove ${ }^{3}$, Tobias Klatte ${ }^{4}$, Kasra Saeb-Parsy ${ }^{2}$, Sarah Howles ${ }^{3}$, Grant D Stewart ${ }^{1}$, Ben Turney ${ }^{3}$, Oliver Wiseman ${ }^{2}$

${ }^{1}$ University of Cambridge \& Cambridge University Hospitals NHS Foundation Trust, UK;

${ }^{2}$ Cambridge University Hospitals NHS Foundation Trust, UK;

${ }^{3}$ Oxford University Hospitals NHS Trust, UK;

${ }^{4}$ University of Cambridge \& Royal Bournemouth and Christchurch Hospitals NHS Foundation Trust, UK

Correspondence: Fanourios Georgiades: fanourios.georgiades@nhs.net

Submitted: June 27, 2020. Accepted: August 7, 2020. Published: September 1, 2020.

\begin{abstract}
\section{Background and objectives}

The coronavirus disease 2019 (COVID-19) pandemic is having a significant impact on healthcare delivery. As a result, management of patients with ureteric stones has likely been affected. We report our study protocol for the investigation of ureteric stone management during and after the COVID-19 pandemic.

\section{Material and methods}

The COVID Stones study is a multicenter national cohort study of the management and outcomes of patients with ureteric stones before, during, and after the COVID-19 pandemic in the United Kingdom. The study will consist of three data collection periods, pre-pandemic ("pre-COVID"), pandemic ("COVID"), and postpandemic ("post-COVID"). This will allow quantification of what "normal" was, how this has changed, and to capture any persisting changes in management. The primary outcome evaluating the success rate of the initial treatment decision will be assessed following a 6-month follow-up from the time of first presentation and will be performed for each recruited patient from each of the three data collection periods. This will allow comparison between both management and outcomes before, during, and after the pandemic.
\end{abstract}

\section{Conclusions}

We anticipate that this study will lead to an increased understanding of the impact of the outcomes of emergency management of ureteric stones following changes in clinical practice due to the COVID-19 pandemic health provision restrictions.

Keywords: COVID-19; clinical management; ureteric stones; urolithiasis 


\section{INTRODUCTION}

\section{Background}

Urinary tract stone disease is a major clinical and economic health burden, affecting up to $20 \%$ of men and $10 \%$ of women by 70 years of age and accounting for over 85,000 hospital episodes per year in the United Kingdom. ${ }^{1,2}$ The estimated annual cost of urinary tract stones managed within the National Health Service (NHS) in England alone is between $£ 190$ million and $£ 324$ million. $^{3}$ The majority of ureteric stones will pass through urine spontaneously ${ }^{4}$; however, some may require fragmentation either via ureteroscopic (URS) laser lithotripsy or extracorporeal shockwave lithotripsy (ESWL). Emergency decompression (via a nephrostomy or stent insertion) is an essential step in management for patients with infected obstructed systems owing to the associated morbidity (sepsis, loss of renal function) and mortality ${ }^{5}$ (Figure 1).

In the United Kingdom, guidelines published by the National Institute for Clinical Excellence (NICE) in $2019^{6}$ recommended that patients over 18 years of age with a ureteric stone measuring $<10 \mathrm{~mm}$ should be offered ESWL as the first-line treatment. URS treatment can be considered if stone clearance is not possible within 4 weeks with ESWL, if there are contraindications for ESWL, if the stone is not targetable with ESWL, or if a previous course of ESWL has failed. For stones measuring $>10 \mathrm{~mm}$ and up to $20 \mathrm{~mm}$, URS should be offered first. ESWL can be considered if local facilities allow for stone clearance within 4 weeks. ${ }^{6}$

Severe acute respiratory syndrome coronavirus 2 (SARS-CoV-2) is a novel coronavirus identified first in the city of Wuhan, capital of the Hubei Province in China, in December 2019. ${ }^{7}$ Infection with this novel coronavirus, termed coronavirus disease-19 (COVID-19), is responsible for significant morbidity and mortality throughout the world, which prompted the World Health Organization to declare COVID-19 a pandemic in March 2020. ${ }^{8}$ This pandemic has resulted

FIG. 1 Patient flow diagram and primary outcome of coronavirus disease (COVID) Stones study. Adult patients with a ureteric calculus can be managed conservatively, with extracorporeal shockwave lithotripsy (ESWL), nephrostomy, ureteroscopic (URS) ( \pm laser lithotripsy \pm stent insertion), or stent insertion. The COVID Stones study will assess the outcome of the primary treatment option.

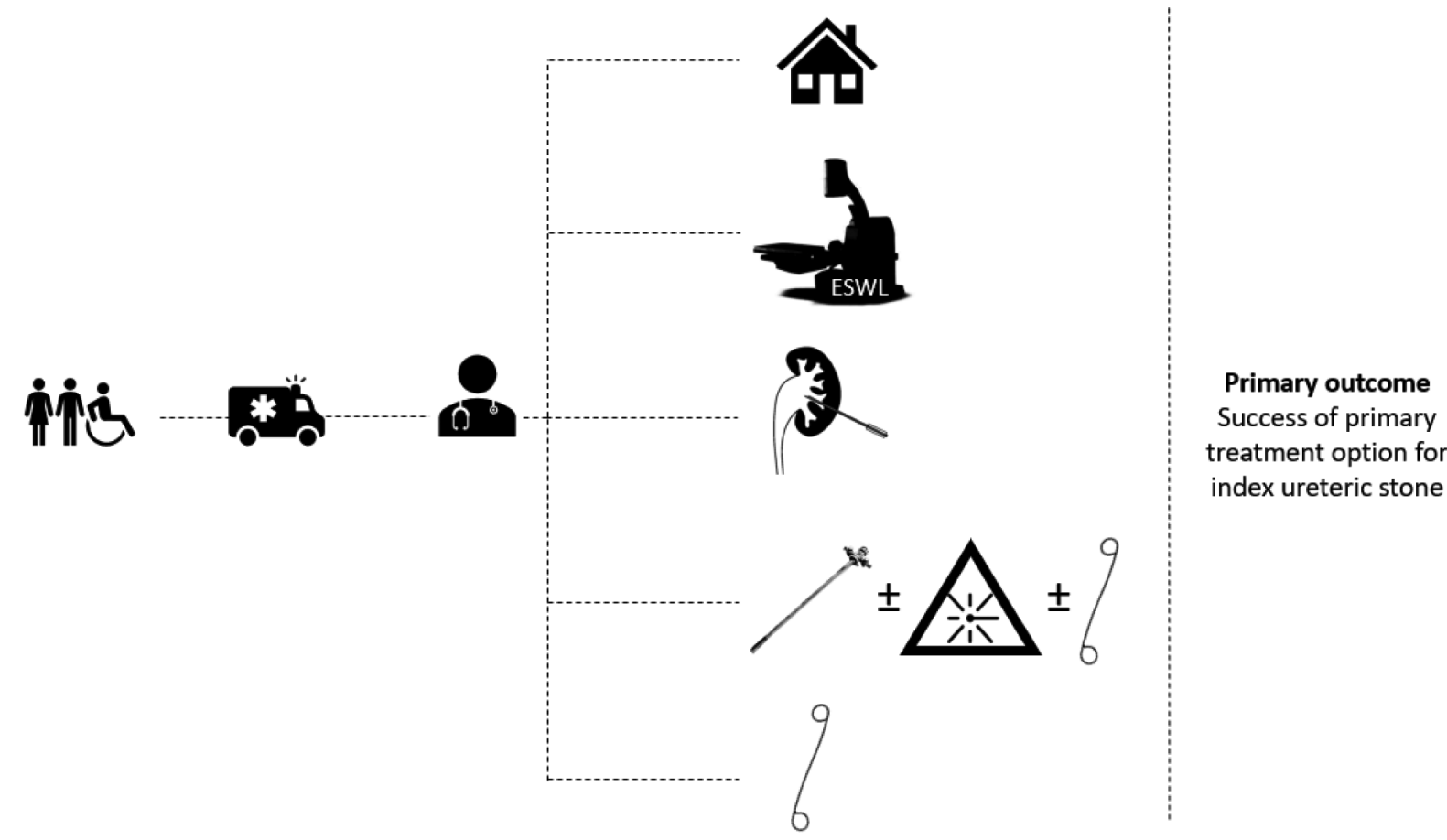

Endolum Endourol Vol 3(3):e22-e28; September 1, 2020.

This article is distributed under the terms of the Creative Commons Attribution-Non

Commercial 4.0 International License. () Georgiades F, et al 
in worldwide disruption to healthcare provision. ${ }^{9}$ Results from a multicenter international study (COVIDSurg) have indicated that perioperative infection with the SARS-CoV-2 virus is associated with an unselected 30 -day mortality of $18.9 \% .{ }^{10}$ These findings, in addition to ongoing pressures on healthcare services, have resulted in the cancellation of many elective procedures and alterations in clinical practice in favor of nonoperative treatment options wherever possible $(9,11)$. Furthermore, during the height of the pandemic in the United Kingdom, attendances at emergency departments with non-COVID-19-related presentations fell dramatically as patients delayed and avoided presentation to hospitals. ${ }^{12}$

\section{Rationale for this study}

Existing literature concerning COVID-19 and endourology details the principles and practicalities of resource reallocation in the context of healthcare service disruption, with emphasis on alterations to clinical care to accommodate the same. However, these articles fail to report on patient outcomes following the proposed changes. Their impact serves to improve consistency in managing patients between different team members and facilitate appropriate use of theater resources; however, they do not inform whether or not changes to practice have been beneficial, neutral, or deleterious in the context of the COVID-19 pandemic.

This study seeks to identify changes in the presentation and management of patients diagnosed with ureteric stones during and after the COVID-19 pandemic when compared to the pre-COVID-19 era. In addition, this study will increase our understanding of the optimal management of individuals with ureteric stones.

\section{METHODS}

\section{Hypotheses}

1. Observation and ESWL are being used more frequently during COVID-19 than previously. This will result in higher rates of failed index intervention and subsequent re-presentation to hospital.

2. There will be an increase in the proportion of patients presenting with elevated creatinine/ acute kidney injury and sepsis due to patients' reluctance to attend emergency services during the pandemic and the subsequent delayed presentation.

3. There will be an increase in procedures performed under local or spinal anesthesia, compared to general anesthetics.

4. There will be a delay in time to initial and follow-up operative management.

\section{Objectives}

To determine how management of ureteric stones has changed during and after the COVID-19 pandemic in the United Kingdom and whether this has affected patient outcomes.

\section{Study design}

The COVID Stones study is a multicenter national cohort study of the management and outcomes of patients presenting with ureteric stones during and after the COVID-19 pandemic in the United Kingdom. The study will consist of three data collection periods, pre-pandemic ("pre-COVID" from 23 March 2019 to 22 June 2019), pandemic (“COVID” from the date of the first COVID-positive admission to the participating site until 3 months from that date), and post-pandemic ("post-COVID" from 23 March 2021 to 22 June 2021). However, for the post-COVID period, the timing may be changed depending on the prevalence of SARS-CoV-2 in the community and the restrictions imposed by the UK government at the time. This will allow quantification of what was practiced prior to the pandemic, how practice has changed, and to capture any persisting changes in management. The data collection periods were selected in such a way so as to avoid introduction of seasonal bias due to variations in ureteric stone presentation.

To assess our study's patient outcomes, a followup of 6 months will be required for each recruited patient; the pertinent data will be collected for every study participant for each of the three data collection periods (Figure 2). This will allow comparison between both management and outcomes before, during, and after the pandemic.

\section{Study population and recruitment}

Adults ( $\geq 18$ years old), presenting to the NHS services with a ureteric stone confirmed by contrast or noncontrast computed tomography (CT) scan of their abdomen, will be included. Patients with multiple 
FIG. 2 Coronavirus disease (COVID) stones patient recruitment periods will capture patients prior, during, and after the COVID-19 pandemic (examples of potential patient pathways shown). Outcomes will be assessed following a 6-month follow-up period from study entry for each participant at each data collection period. *Dates for the post-COVID period are subject to change, depending on the prevalence of COVID-19 during that period in the United Kingdom.

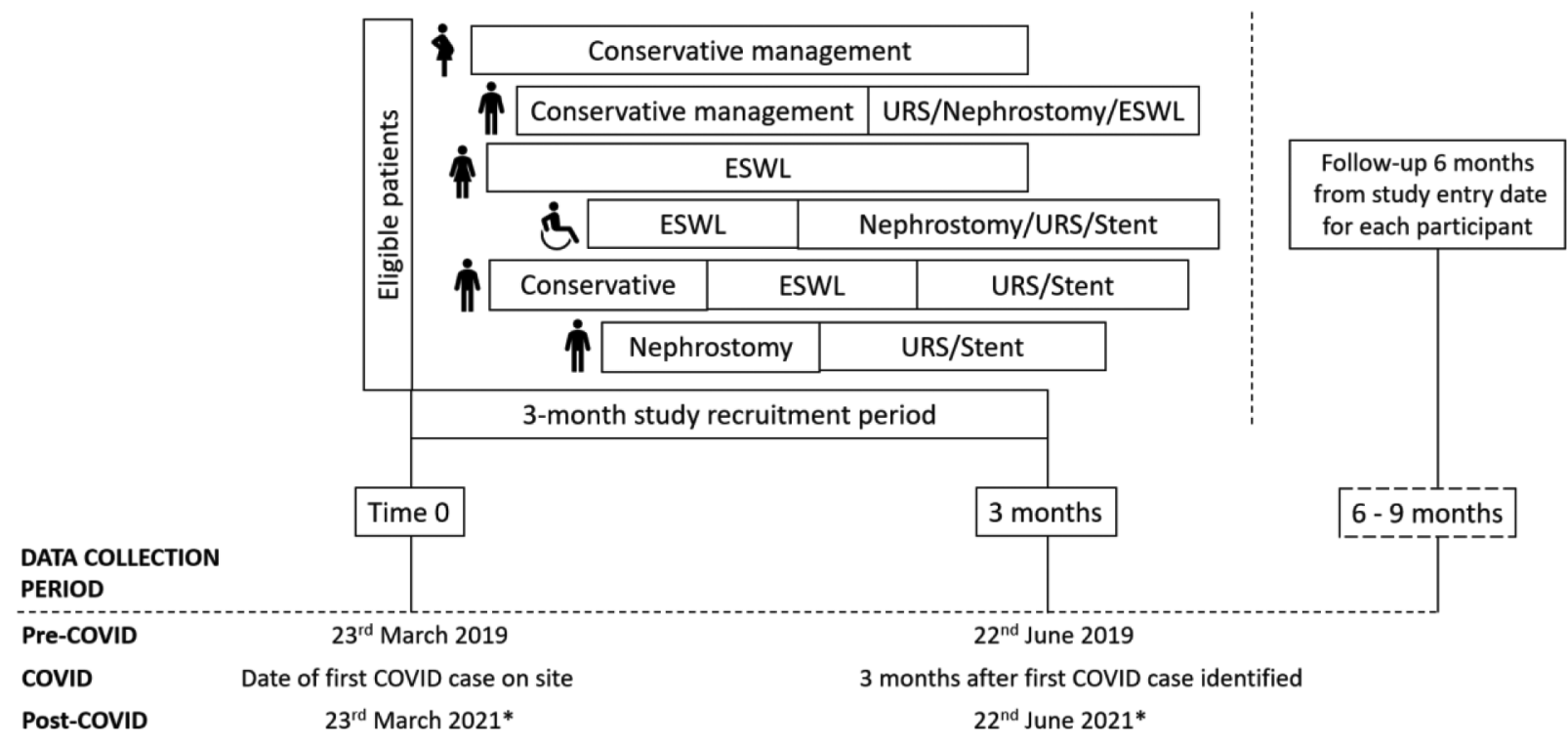

URS = ureteroscopic; ESWL = extracorporeal shockwave lithotripsy.

stones will be eligible provided a stone was identified within the ureter. Patients will be identified by completing a retrospective review of all abdominal CT scans undertaken during the study data collection periods at each participating site.

\section{Inclusion criteria}

- Any adult ( $\geq 18$ years) in the United Kingdom

- Ureteric stone(s) identified on CT scan

\section{Exclusion criteria}

- Patients $<18$ years of age at the time of stone disease diagnosis

- Nonureteric stone disease only (e.g., bladder stone(s) only or renal stone(s) only)

\section{Outcome measures}

\section{Primary outcome measure}

Success of primary treatment modality determined by no additional treatment required for index ureteric stone.

\section{Secondary outcome measures}

Secondary outcomes will look at morbidity and mortality of patients with stone disease. The following outcomes will be compared between each time period:

- Evaluation of rates of:

- Nonoperative management

- ESWL

- Operative rate

- JJ stent insertion

- URS and laser lithotripsy

- Percutaneous nephrolithotomy (PCNL)

- Nephrostomy insertion

- Hospital admission rates

- Length of time to operative management from first presentation

- Pulmonary complication rate

- Admission to critical care (Level 2 or 3 care)

- 30-day and 6-month mortality rate

- Length of hospital stay

- Readmission rate within 6 months 
- Type of anesthesia for operative management options

- Impact of index stone on baseline renal function

- Complication rate if operative management was required

- Adherence to NICE guidelines

\section{Data collection and processing}

Data from eligible patients at each site will be collected by local principal investigators (PIs) and collaborators for each time period. The Research Electronic Data Capture (REDCap) online software will be used as the platform of choice to collect and store data. ${ }^{13,14}$ The REDCap server will be managed and hosted by the University of Oxford, the United Kingdom, for this study. Access to the REDCap server will be given to the registered PI of each site. They will upload the collected data to the server with the assistance of up to four other local collaborators.

The data collected will include key demographic details, management details, and outcomes following a follow-up period of 6 months since study entry for each participating patient for each of the three time periods (Figure 2). Data collected will be compared with NICE guidelines on assessment and management of ureteric stones. Collection of additional data will facilitate identification of confounding factors to prevent bias.

\section{Data analysis plan}

The study will be reported according to the STROBE guidelines for observational studies. Data will be retrieved from the REDCap Server at 6 months following the end of the post-COVID data collection period (estimated to be in December 2021). The data will mainly be presented as descriptive statistics, and comparisons between groups for each time period will be performed. Data will not be compared between individual sites. Multivariable logistic regression will be used to identify predictors of treatment success of the index intervention.

\section{Dissemination and expected outputs}

At a minimum, we anticipate this study to be published in a peer-reviewed medical or scientific journal. This project will be submitted for presentation at national and international conferences. All participating individuals will be listed as collaborators and be part of the group authorship under the collective title, "The COVID Stones Collaborative."

\section{DISCUSSION}

An adaptive and evidence-based response is vital to lessen the impact of the COVID-19 pandemic on current and future urological practices and patient outcomes, as well as to inform us about the management strategies for future pandemics.

There has been an increase in collaborative research studies during the COVID-19 pandemic. Some studies have focused on patient outcomes following SARS$\mathrm{CoV}-2$ infection, such as COVIDSurg, investigating patient outcomes following surgery and perioperative SARS-CoV-2 infection. ${ }^{10}$ The same group of investigators and collaborators is also coordinating an international multicenter study investigating the impact of the COVID-19 pandemic on cancer diagnosis and treatment. The study so far has recruited more than 35,000 patients from more than 1000 centers around the world, and it is anticipated that the initial results will be published at the beginning of August 2020. Other studies have looked at change in practice and associated outcomes of diseases such as appendicitis and cholecystitis. The COVID HAREM (Had Appendicitis Recovered/Recurred Emergency Morbidity/Mortality) study is a UK-wide initiative, which aims to assess adult acute appendicitis during COVID-19. This collaborative published their interim data of 500 patients in July 2020, which demonstrated that conservative management of appendicitis was successful in 90\% of cases during the pandemic. ${ }^{15}$ The COVID HAREM study has recruited more than 3000 patients to date, and their final results are anticipated in September 2020. The CHOLECOVID Collaborative has initiated an international multicenter retrospective cohort study for investigating the impact of the COVID-19 pandemic on managing cholecystitis patients; to date, more than 1500 patients have been recruited for the study from around the world. ${ }^{16}$

Proietti et al. have provided an overview, which suggests strategies for triaging the surgical priority and risk of COVID-19 in patients presenting with urological pathology, for discerning appropriate 
timescales and locations for medical attention in this cohort. ${ }^{17}$ The narrative includes a recommendation to conservatively manage renal colic as far as possible to "avoid admission to an overwhelmed emergency department" and to select patients for endourological surgery according to surgical priority. Metzler et al. mirrored the considerations of Proietti et al. in prioritizing cases according to patient factors (symptoms, comorbidities, renal tract abnormalities, and the presence of a temporizing stent or nephrostomy) and stone factors (unilateral or bilateral obstruction, presence of infection, failure of conservative management). ${ }^{17,18}$

Multiple sources indicate that for patients with an infected and/or obstructed kidney, a ureteric stent, placed under a local anesthetic, should be the intervention of choice, and nephrostomy would be a second-line alternative in the COVID-19 setting. ${ }^{17,19}$ Existing patients awaiting elective intervention of kidney stone disease should be triaged, balancing patient history with the burden of medical emergencies within the healthcare system. Those with stents and those deemed to be at a greater risk of infection therefore ought to be prioritized in this process. ${ }^{17-19}$ Further suggested alterations to practice include maximizing the use of local-anesthetic procedures to avoid exposure to ventilators and one-off day case interventions to reduce healthcare exposures. That said, urologists at the University of Washington expressed preference for URS management of ureteric stones over ESWL given the higher stone-free rates and the reduced need for re-intervention. ${ }^{18}$ It is worth noting that in the United States, the standard of care is for ESWL to be performed under a general anesthetic.

The European Association of Urology Section of Urolithiasis (EULIS) sought to identify how 473 patients who underwent interventions for urinary tract calculi at 11 centers in five countries were affected by the COVID-19 pandemic. ${ }^{20}$ Multiple stones were identified in $21.9 \%$ patients, $23 \%$ underwent emergency intervention, $34.5 \%$ of which were URS stone extractions. In European centers, there was a shift in practice favoring more conservative management. This may have been to avoid definitive measures that require general anesthetic or to assign hospital resources for COVID-19 purposes. The study did not include sufficient follow-up to consider the effect on patient outcomes that this change of practice may have had. ${ }^{18}$ Flammia et al. described alterations in emergency care for kidney stone disease over 6 weeks in a hospital managing the majority of COVID-19 cases in Italy and compared trends with historical data of 12 months from the same center. ${ }^{21}$ They reported no change in the gross number of patients presenting within each time frame, although they noted a higher serum creatinine level in their COVID-19 cohort. This was used as a surrogate marker to suggest delayed presentation of patients to hospital in the context of the pandemic. ${ }^{21}$ There were no changes in diagnostic or therapeutic approaches to emergency management of ureteric stones though, and as with the study by Gokce et al., there was no follow-up to report patient outcomes. ${ }^{20,21}$

We anticipate that this study will increase the understanding of the impact on patient outcomes of changes in clinical practice brought about by the COVID-19 pandemic with regard to the emergency management of ureteric stones.

\section{GRANT SUPPORT}

This study did not receive any funding.

\section{REFERENCES}

1. Heers H, Turney BW. Trends in urological stone disease: A 5-year update of hospital episode statistics. BJU Int 2016;118(5):785-9. http://dx.doi.org/10.1111/bju.13520

2. Scales CD Jr, Smith AC, Hanley JM, Saigal CS; Urologic Diseases in America Project. Prevalence of kidney stones in the United States. Eur Urol 2012;62(1):160-5. http://dx.doi.org/10.1016/j.eururo.2012.03.052

3. Geraghty RM, Cook P, Walker V, Somani BK. Evaluation of the economic burden of kidney stone disease in the UK: A retrospective cohort study with a mean follow-up of 19 years. BJU Int 2020;125(4):586-94. http://dx.doi.org/10.1111/bju.14991

4. Shah TT, Gao C, Peters M, et al. Factors associated with spontaneous stone passage in a contemporary cohort of patients presenting with acute ureteric colic: Results from the multi-centre cohort study evaluating the role of inflammatory markers in patients presenting with acute ureteric Colic (MIMIC) study. BJU Int 2019;124(3):504-13. http://dx.doi.org/10.1111/ bju. 14777

5. Pearle MS, Pierce HL, Miller GL, et al. Optimal method of urgent decompression of the collecting system for

Endolum Endourol Vol 3(3):e22-e28; September 1, 2020.

This article is distributed under the terms of the Creative Commons Attribution-Non

Commercial 4.0 International License. () Georgiades F, et al 
obstruction and infection due to ureteral calculi. J Urol 1998;160(4):1260-4. http://dx.doi.org/10.1016/ S0022-5347(01)62511-4

6. NICE guideline NG118: Renal and ureteric stones: Assessment and management. 2019. https://www.nice. org.uk/guidance/ng118 (accessed on: July 16, 2020).

7. Zhou F, Yu T, Du R, et al. Clinical course and risk factors for mortality of adult inpatients with COVID-19 in Wuhan, China: A retrospective cohort study. Lancet 2020;395:1054-62. http://dx.doi.org/10.1016/ S0140-6736(20)30566-3

8. Cucinotta D, Vanelli M. WHO declares COVID-19 a pandemic. Acta Biomed 2020;91:157-60.

9. COVIDSurg Collaborative. Elective surgery cancellations due to the COVID-19 pandemic: Global predictive modelling to inform surgical recovery plans [published online ahead of print, 2020 May 12]. Br J Surg 2020. http://dx.doi.org/10.1002/bjs.11746

10. COVIDSurg Collaborative. Mortality and pulmonary complications in patients undergoing surgery with perioperative SARS-CoV-2 infection: An international cohort study [published correction appears in Lancet 2020 Jun 9]. Lancet 2020;396(10243):27-38. http:// dx.doi.org/10.1016/S0140-6736(20)31182-X

11. Royal College of Surgeons of England. Updated Intercollegiate General Surgery Guidance on COVID-19. London: Royal College of Surgeons of England, 2020. https://www.rcseng.ac.uk/coronavirus/joint-guidancefor-surgeons-v2/ (accessed on: July 17, 2020).

12. Thornton J. Covid-19: A\&E visits in England fall by 25\% in week after lockdown. BMJ 2020;369:m1401. http://dx.doi.org/10.1136/bmj.m1401

13. Harris PA, Taylor R, Thielke R, et al. Research electronic data capture (REDCap) - A metadata-driven methodology and workflow process for providing translational research informatics support. J Biomed Inform 2009;42(2):377-81. http://dx.doi.org/10.1016/j. jbi.2008.08.010
14. Harris PA, Taylor R, Minor BL, et al. The REDCap consortium: Building an international community of software platform partners. J Biomed Inform 2019;95:103208. http://dx.doi.org/10.1016/j.jbi.2019.103208

15. Javanmard-Emamghissi H, Boyd-Carson H, Hollyman $\mathrm{M}$, et al. The management of adult appendicitis during the COVID-19 pandemic: An interim analysis of a UK cohort study [published online ahead of print, 2020 Jul 15]. Tech Coloproctol 2020;1-11. http://dx.doi. org/10.1007/s10151-020-02297-4

16. CHOLECOVID Collaborative. CHOLECOVID study protocol. 2020. https://cholecovid.org/ (accessed on: July 21, 2020).

17. Proietti S, Gaboardi F, Giusti G. Endourological stone management in the era of the COVID-19 [published online ahead of print, 2020 Apr 14]. Eur Urol 2020;S0302-2838(20)30217-7. http://dx.doi. org/10.1016/j.eururo.2020.03.042

18. Metzler IS, Sorensen MD, Sweet RM, Harper JD. Stone care triage during COVID-19 at the University of Washington. J Endourol 2020;34(5):539-40. http:// dx.doi.org/10.1089/end.2020.29080.ism

19. Stensland KD, Morgan TM, Moinzadeh A, et al. Considerations in the triage of urologic surgeries during the COVID-19 pandemic. Eur Urol 2020;77(6):663-6. http://dx.doi.org/10.1016/j.eururo.2020.03.027

20. Gökce Mi, Yin S, Sönmez MG, et al. How does the COVID-19 pandemic affect the preoperative evaluation and anesthesia applied for urinary stones? EULIS eCORE-IAU multicenter collaborative cohort study. Urolithiasis 2020;48(4):345-51. http://dx.doi. org/10.1007/s00240-020-01193-8

21. Flammia S, Salciccia S, Tufano A, et al. How urinary stone emergencies changed in the time of COVID-19? [published online ahead of print, 2020 May 28]. Urolithiasis 2020;1-3. http://dx.doi.org/10.1007/ s00240-020-01198-3

Endolum Endourol Vol 3(3):e22-e28; September 1, 2020.

This article is distributed under the terms of the Creative Commons Attribution-Non Commercial 4.0 International License. (C) Georgiades F, et al 